\title{
Autophagy inhibition-mediated epithelial-mesenchymal transition augments local myofibroblast differentiation in pulmonary fibrosis
}

\author{
Charlotte Hill', Juanjuan Li ${ }^{1}$, Dian Liu², Franco Conforti, ${ }^{3,4}$, Christopher J. Brereton ${ }^{3,4}$, Liudi Yao', Yilu Zhou', \\ Aiman Alzetani ${ }^{4,5}$, Serena J. Chee ${ }^{5,6}$, Ben G. Marshall $\mathbb{1}^{4,5}$, Sophie V. Fletcher ${ }^{4,5}$, David Hancock

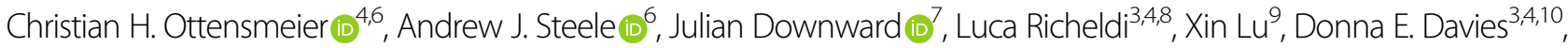 \\ Mark G. Jones (1) $3,4,10$ and Yihua Wang (10)
}

\begin{abstract}
Idiopathic pulmonary fibrosis (IPF), the prototypic progressive fibrotic interstitial lung disease, is thought to be a consequence of repetitive micro-injuries to an ageing, susceptible alveolar epithelium. Ageing is a risk factor for IPF and incidence has been demonstrated to increase with age. Decreased (macro)autophagy with age has been reported extensively in a variety of systems and diseases, including IPF. However, it is undetermined whether the role of faulty autophagy is causal or coincidental in the context of IPF. Here, we report that in alveolar epithelial cells inhibition of autophagy promotes epithelial-mesenchymal transition (EMT), a process implicated in embryonic development, wound healing, cancer metastasis and fibrosis. We further demonstrate that this is attained, at least in part, by increased p62/SQSTM1 expression that promotes p65/RELA mediated-transactivation of an EMT transcription factor, Snail2 (SNAI2), which not only controls EMT but also regulates the production of locally acting profibrogenic mediators. Our data suggest that reduced autophagy induces EMT of alveolar epithelial cells and can contribute to fibrosis via aberrant epithelial-fibroblast crosstalk.
\end{abstract}

\section{Introduction}

Idiopathic pulmonary fibrosis (IPF) is the most common type of chronic, progressive fibrotic interstitial lung disease. It occurs with comparable incidence to that of stomach, brain and testicular cancer $^{1}$, and the median survival of patients with IPF is only 3 years $^{2,3}$. IPF is characterised by aberrant extracellular matrix (ECM) deposition, which leads to decreased lung compliance,

Correspondence: Yihua Wang (yihua.wang@soton.ac.uk)

${ }^{1}$ Biological Sciences, Faculty of Environmental and Life Sciences, University of Southampton, Southampton SO17 1BJ, UK

${ }^{2}$ Department of Oncology, Tongji Hospital, Tongji Medical College, Huazhong University of Science and Technology, Wuhan, China

Full list of author information is available at the end of the article.

These authors contributed equally: Charlotte Hill, Juanjuan Li

Edited by B. Zhivotovsky disrupted gas-exchange and ultimately respiratory failure and death. As approved therapies only slow disease progression there is significant unmet medical need.

Evidence suggests that interacting genetic and environmental factors are crucial for the development of IPF, with repetitive injuries to aged alveolar epithelium thought to play an important role ${ }^{2}$. Ageing is a risk factor for IPF, with disease incidence increasing with age $^{3}$. Decreased (macro)autophagy with age has been reported extensively in a variety of systems and diseases ${ }^{4}$, including $\mathrm{IPF}^{5-9}$. Autophagy is a tightly controlled, evolutionarily conserved process for the lysosomal degradation of cytoplasmic cargo, long-lived proteins and organelles. Growing evidence suggests that autophagy acts as an

\section{(c) The Author(s) 2019}

(c) Open Access This article is licensed under a Creative Commons Attribution 4.0 International License, which permits use, sharing, adaptation, distribution and reproduction cc. in any medium or format, as long as you give appropriate credit to the original author(s) and the source, provide a link to the Creative Commons license, and indicate if changes were made. The images or other third party material in this article are included in the article's Creative Commons license, unless indicated otherwise in a credit line to the material. If material is not included in the article's Creative Commons license and your intended use is not permitted by statutory regulation or exceeds the permitted use, you will need to obtain permission directly from the copyright holder. To view a copy of this license, visit http://creativecommons.org/licenses/by/4.0/. 
adaptive response to facilitate cell survival and limit cell death following exposure to stressful stimuli ${ }^{10}$.

Tissues from IPF patients are characterised by defective autophagy responses ${ }^{5-8}$, although it is unclear whether faulty autophagy exerts an effect directly, or if this change is merely coincident with IPF. Loss of autophagy-related protein 7 (ATG7), a ubiquitin-activating enzyme that is essential for autophagy, has been demonstrated both in vivo and in vitro to induce endothelial-to-mesenchymal transition (EndMT). Autophagy inhibition, by loss of ATG7, has been associated with changes in endothelial cell (EC) architecture, reduced endothelial markers and increased mesenchymal markers, whilst EC-specific Atg7 knockout mice had increased susceptibility to bleomycin induced-fibrosis ${ }^{11}$. Furthermore, inhibition of autophagy is sufficient to induce acceleration of both bronchial epithelial cell senescence and differentiation of lung fibroblasts into myofibroblasts ${ }^{8}$. Here, we report that autophagy inhibition promotes epithelial-mesenchymal transition (EMT) in alveolar epithelial cells. EMT is a reversible biological process where epithelial cells gain migratory and invasive abilities through loss of cell polarity and cadherin-mediated cell-cell adhesion; this process has been implicated in embryonic development, wound healing, cancer metastasis and organ fibrosis ${ }^{12}$. Specific transcription factors, EMT transcription factors (EMT-TFs), including Snail, ZEB and TWIST are able to promote the repression of epithelial features and induction of mesenchymal features ${ }^{13,14}$. We show that promotion of EMT by autophagy inhibition in alveolar epithelial cells is attained, at least in part, by increased p62/SQSTM1 expression that induces p65/RELA mediated-transactivation of Snail2 (SNAI2), which not only controls EMT but also regulates the production of locally acting profibrogenic mediators. This suggests that autophagy inhibition-induced EMT of alveolar epithelial cells contributes to fibrosis not only by affecting the epithelial phenotype but also via aberrant epithelial-fibroblast crosstalk.

\section{Results}

\section{Autophagic activity is downregulated in IPF lungs}

Defective autophagy responses in tissues from IPF patients have been reported previously ${ }^{6,8}$. Similarly, we observed evidence of autophagy inhibition as determined by $\mathrm{p} 62 / \mathrm{SQSTM} 1$ accumulation in lung epithelial cells in IPF samples (Fig. 1; Supplementary Fig. S1a). p62/ SQSTM1 is a key protein involved in autophagy that recognises cellular waste, which is then sequestered and degraded by autophagy ${ }^{15}$. A strong staining for p62/ SQSTM1 was detected in IPF tissue within epithelial cells of thickened alveoli septae where collagen deposition in the interstitium was demonstrated (Fig. 1b; Supplementary Fig. S1a), as well as within fibroblastic foci (Fig. 1a). In contrast, there was weak p62/SQSTM1 expression and little collagen deposition detected in alveoli of control lung tissue (Fig. 1c). Using a publicly available dataset ${ }^{16}$, the mRNA level of SQSTM1 (p62) in IPF epithelial cells was evaluated. It was found that SQSTM1 (p62) mRNA expression level was reduced in IPF lungs (Supplementary Fig. S1b) whilst its protein level as assessed by immunohistochemistry was increased in IPF (Fig. 1b; Supplementary Fig. S1a). Given that p62/SQSTM1 protein levels are predominantly regulated by autophagic activity ${ }^{17}$, these results suggest that autophagic activity is downregulated in IPF epithelial cells.

\section{Alteration of autophagic activity affects cellular plasticity of alveolar epithelial cells}

To investigate its role in alveolar epithelial cells, autophagic activity was first modified using chemical inhibitors. We treated human alveolar epithelial type II (ATII) cells ${ }^{18-20}$ with an autophagy inhibitor, hydroxychloroquine (HCQ), which is a lysosomotropic autophagy inhibitor. HCQ treatment of ATII cells inhibited autophagic activity, demonstrated by the accumulation of p62/ SQSTM1 (Fig. 2a), inhibition of lysosomal degradation by HCQ also caused accumulation of LC3-II (Fig. 2a). Autophagy inhibition with HCQ resulted in a downregulation of E-cadherin in a time-dependent manner (Fig. 2a). Morphology changes were also observed following HCQ treatment with ATII cells developing an elongated mesenchymal cell phenotype; this was accompanied by actin cytoskeleton reorganisation demonstrated using filamentous actin (F-actin) staining with Phalloidin (Fig. 2c), suggesting induction of a cellular reprogramming process, EMT. A group of transcription factors, termed EMT-TFs, are capable of inducing EMT, these include Snail1 (SNAI1), Snail2 (SNAI2), TWIST1, ZEB1 and ZEB2 ${ }^{13}$, which act by binding E-boxes in the proximal promoter of the $C D H 1$ (E-cadherin) gene to repress its expression. We thus investigated the impact of $\mathrm{HCQ}$ treatment on the mRNA expression levels of E-cadherin $(C D H 1)$ and EMT-TFs in ATII cells. Following HCQ treatment of ATII cells, we observed a downregulation of E-cadherin (CDH1), increased levels of a mesenchymal marker Vimentin (VIM) and an EMT-TF Snail2 (SNAI2), but not others (Fig. 2b).

To confirm our observations with HCQ, we treated ATII cells with another autophagy inhibitor, Bafilomycin-A1 (Baf-A1), which inhibits autophagy by disrupting vesicular acidification leading to an accumulation of mature, undigested autophagosomes ${ }^{21}$. Autophagy inhibition by Baf-A1 treatment of ATII cells resulted in a similar induction of EMT, evidenced by a reduction in E-cadherin (Supplementary Fig. S2a), an increase of Vimentin (VIM) (Supplementary Fig. S2b), and an upregulation of EMT-TF Snail2 (SNAI2) (Supplementary Fig. S2b). Morphology 


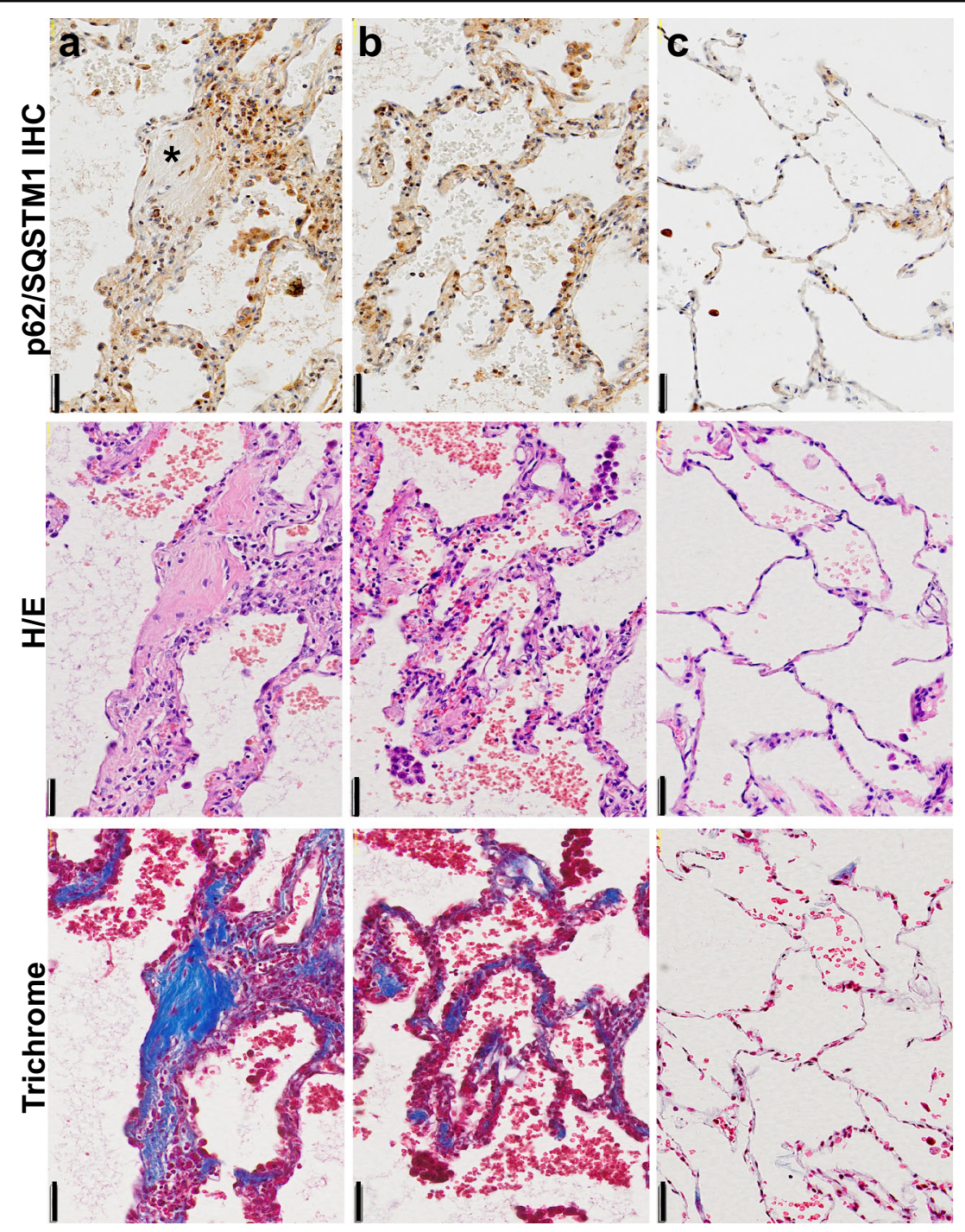

Fig. 1 p62/SQSTM1 is highly expressed in IPF fibroblastic foci and epithelial cells of thickened alveoli septae where collagen deposition in the interstitium is also evident. Serial sections of IPF (fibroblastic foci in (a), and epithelial cells of thickened alveoli septae in (b)) or control lung tissue (c) were stained for p62/SQSTM1 (top panel), with H\&E (middle panel) or Masson's trichrome stain (bottom panel, collagen shown in blue). $n=$ 3. *a fibroblastic focus. Scale bars: $50 \mu \mathrm{m}$

changes were observed following Baf-A1 treatment of ATII cells, with cells developing invasive phenotype when cultured in Matrigel (Supplementary Fig. S2c).

We further used small interfering RNAs (siRNAs) against autophagy-related protein 5 (ATG5) to study the effects of inhibition of autophagic activity in ATII cells. ATG5, a ubiquitin-protein ligase (E3)-like enzyme, is essential for autophagy due to its role in autophagosome elongation. ATG5 siRNA-transfection of ATII cells inhibited autophagic activity, evidenced by the accumulation of p62/SQSTM1 (Fig. 3a, c). As a result, ATG5 depletion led to a downregulation of E-cadherin at both the mRNA and protein levels (Fig. 3a, b), an increase in VIM (Vimentin) at mRNA level (Fig. 3b), and a rearrangement of the F-actin cytoskeleton (Fig. 3c). Similar results were observed in A549 alveolar epithelial cells where autophagy inhibition by ATG5 depletion down regulated E-cadherin levels and increased Snail2 (SNAI2) expression (Supplementary Fig. S3a-c).

In a wound healing context, the EMT process allows epithelial cells to adopt a more migratory mesenchymal phenotype in order to spread rapidly and cover the wounded $\operatorname{area}^{22}$. Therefore, a wound scratch assay was utilised to test whether ATG5 depletion using ATG5 siRNA was sufficient to augment cell mobility. This showed that 20 hours after creating the scratch wound, ATG5 siRNA-transfected ATII cells had more completely repaired the wound compared to control 

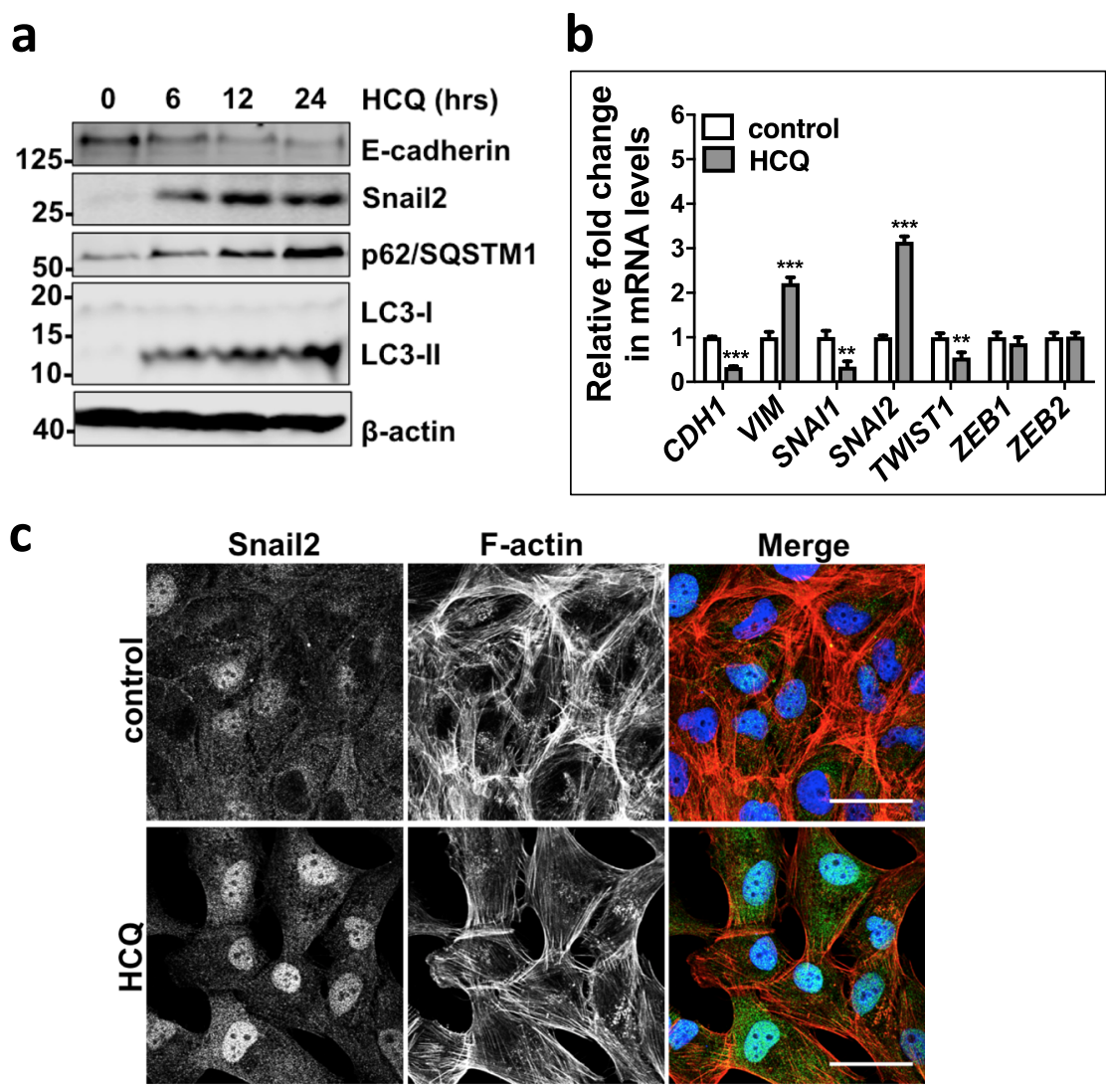

Fig. 2 Autophagic activity altered by chemicals affects cellular plasticity of ATII cells. a Protein expression of E-Cadherin, Snail2, p62/SQSTM1 and LC3 in ATIl cells treated with HCQ $(25 \mu \mathrm{M})$ at indicated times. $\beta$-actin was used as a loading control. b Fold change in mRNA levels of CDH1 (Ecadherin), VIM (Vimentin), SNAI1 (Snail1), SNAI2 (Snail2), TWIST1, ZEB1 and ZEB2 in ATIl cells cultured in the absence or presence of HCQ (25 $\mu M$ ) for 24 hours. GAPDH-normalised mRNA levels in control cells were used to set the baseline value at unity. Data are mean \pm s.d. $n=3$ samples per group. ${ }^{* * *} P<0.001$. c Immunofluorescence staining of Snail2 (green) and F-actin (red) in ATIl cells cultured in the absence or presence of HCQ (25 $\left.\mu \mathrm{M}\right)$ for $24 \mathrm{~h}$. Rhodamine-phalloidin was used to stain F-actin. DAPI (blue) was used to stain nuclei. Scale bar: $40 \mu \mathrm{m}$

siRNA-transfected cells (Fig. $3 \mathrm{~d} ; \mathrm{P}<0.001$ ). In addition, we found in both the Transwell migration (Fig. 3e) and Matrigel invasion assays (Supplementary Fig. S3d), knockdown of ATG5 promoted cell migration and invasion respectively.

Having examined the effects of autophagy inhibition in ATII cells, we next evaluated the effect of autophagy induction using Rapamycin, which inhibits mammalian Target Of Rapamycin (mTOR) ${ }^{23}$. As predicted, following Rapamycin treatment of ATII cells, phosphorylation levels of mTOR were reduced (Supplementary Fig. S4a), and autophagy activity was induced, indicated by a downregulation of p62/SQSTM1 and an increase in LC3-II (Supplementary Fig. S4a). We also found an increase in CDH1 (E-cadherin), and reductions in VIM (Vimentin) and SNAI2 (Snail2) mRNA levels in ATII cells treated with Rapamycin (Supplementary Fig. S4b), and these effects were mainly via the induction of autophagy, since knockdown of ATG5 completely abolished the changes (Supplementary Fig. S4b).
Together, our results demonstrate that autophagic activity is an important regulator of cellular plasticity in alveolar epithelial cells.

\section{Autophagy inhibition induces EMT via p62/SQSMT1-NFKB- Snail2 pathway in alveolar epithelial cells}

We recently reported that in colorectal and pancreatic cancer cells, inhibition of autophagy induces EMT via p62/SQSMT1-NFkB pathway ${ }^{24}$. Therefore, we investigated if this pathway is involved in initiating autophagy inhibition-induced EMT in ATII cells. Comparison of control and HCQ-treated cells revealed that p65/RELA accumulated in the nuclei of treated cells (Fig. 4a), indicating that the NF- $\mathrm{KB}$ pathway is activated in ATII cells when autophagy is inhibited. Functionally, p62/SQSTM1 or p65/RELA knockdown abolished the increase in Snail2 following autophagy inhibition by HCQ treatment in ATII cells (Fig. 4b). Similar results were observed in A549 cells (Supplementary Fig. S5). While knockdown of ATG5 induced EMT, as evidenced by a reduction in $\mathrm{CDH} 1$ 

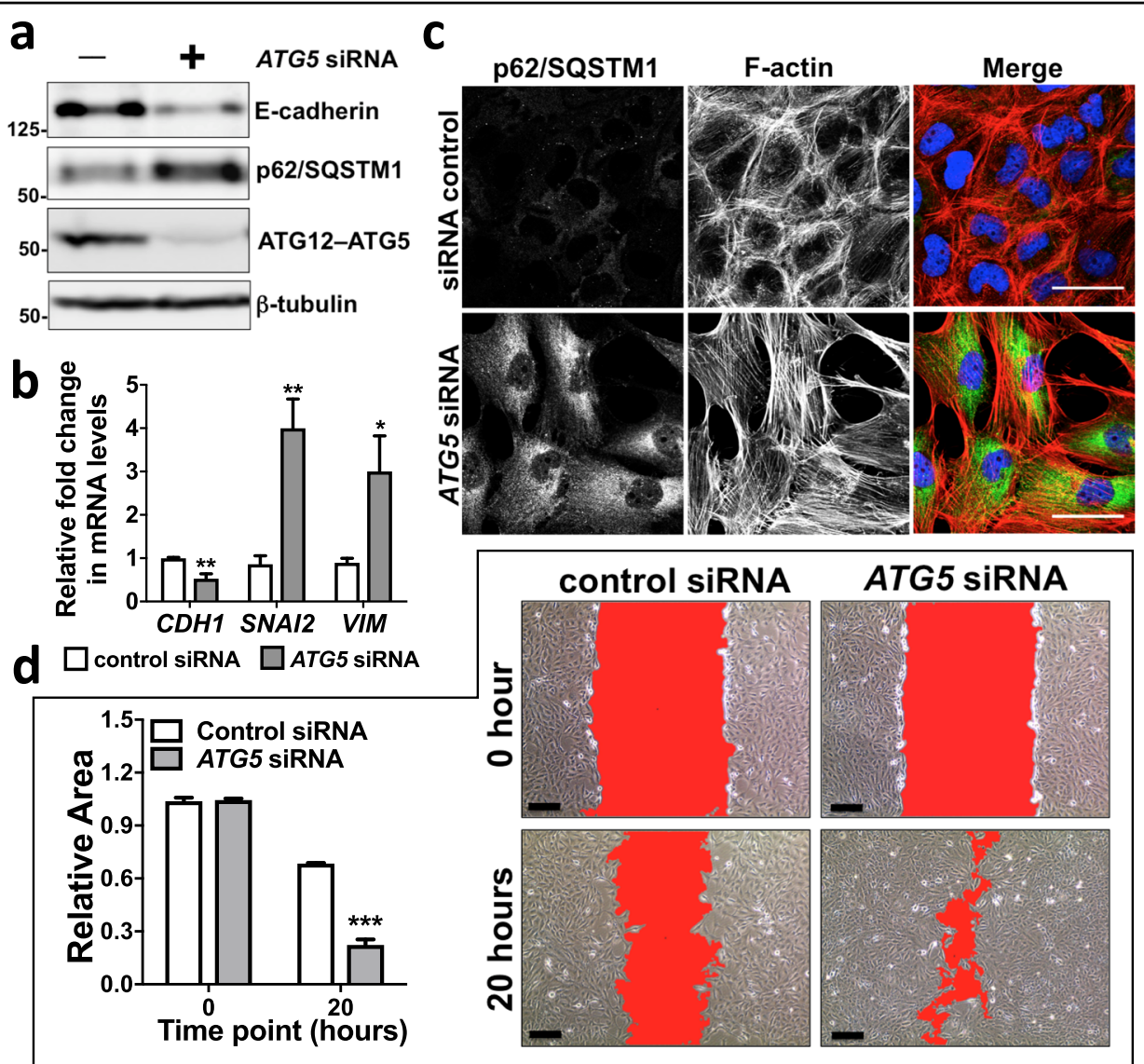

$\mathbf{e}$

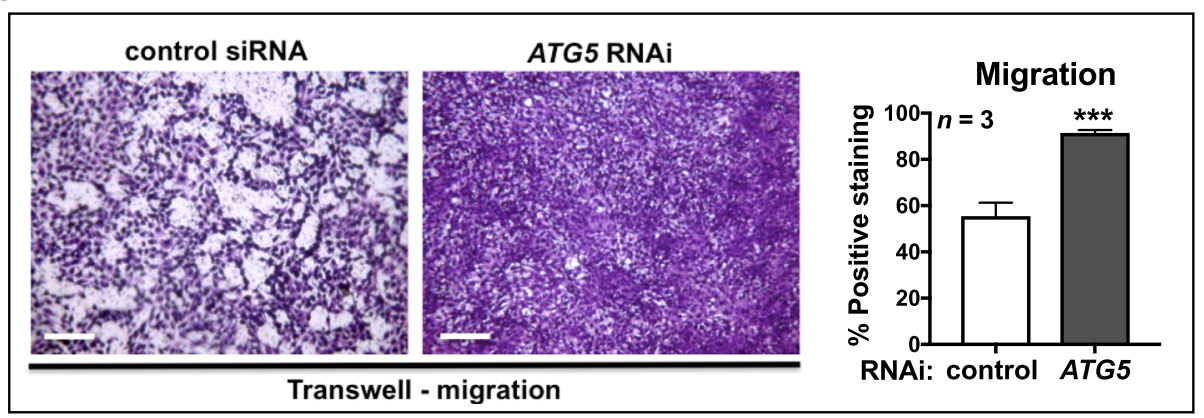

Fig. 3 Autophagy inhibition by ATG5 depletion induces EMT and cell migration in ATIl cells. a Protein expression of E-cadherin, p62/SQSTM and ATG12-ATG5 in ATII cells transfected with indicated siRNAs. $\beta$-tubulin was used as a loading control. $\mathbf{b}$ Fold change in the mRNA levels of CDH1 (E-cadherin), SNAI2 (Snail2) and VIM (Vimentin) in ATII cells transfected with indicated siRNAs. GAPDH-normalised mRNA levels in control cells were used to set the baseline value at unity. Data are mean \pm s.d. $n=3$ samples per group. ${ }^{*} P<0.01$. c Immunofluorescence staining of p62/SQSTM (green) and F-actin (red) in ATII cells transfected with control or ATG5 siRNAs. Rhodamine-phalloidin was used to stain F-actin. DAPI (blue) was used to stain nuclei. Scale bar: $40 \mu \mathrm{m}$. d Scratch wound assay of ATII cells transfected with control or ATG5 siRNAs. Representative images of ATII cells with the indicated treatments at time 0 or $20 \mathrm{~h}$ after the scratch wound. Wounds have been artificially coloured red to aid visualisation. Scale bar: $200 \mu \mathrm{m}$. The graph shows the area of a wound evaluated with ImageJ, and data are mean \pm s.d. $n=3$. ${ }^{* * *} P<0.001$. e Transwell migration assays in control or ATG5-depleted ATIl cells. Cells were stained with crystal violet. Scale bar: $100 \mu \mathrm{m}$. Data are mean \pm s.d. $n=3$. ${ }^{* * *} P<0.001$

(E-cadherin) mRNA levels and increased SNAI2 (Snail2) mRNA levels, subsequent depletion of RELA (p65) or SQSTM1 (p62) (Supplementary Fig. S5a) partially or completely abolished the increase in SNAI2 (Snail2), and restored $C D H 1$ (E-cadherin) expression (Supplementary Fig. S5b). These data suggest that autophagy inhibition in alveolar epithelial cells promotes EMT via the p62/ SQSMT1-NFKB-Snail2 pathway. 


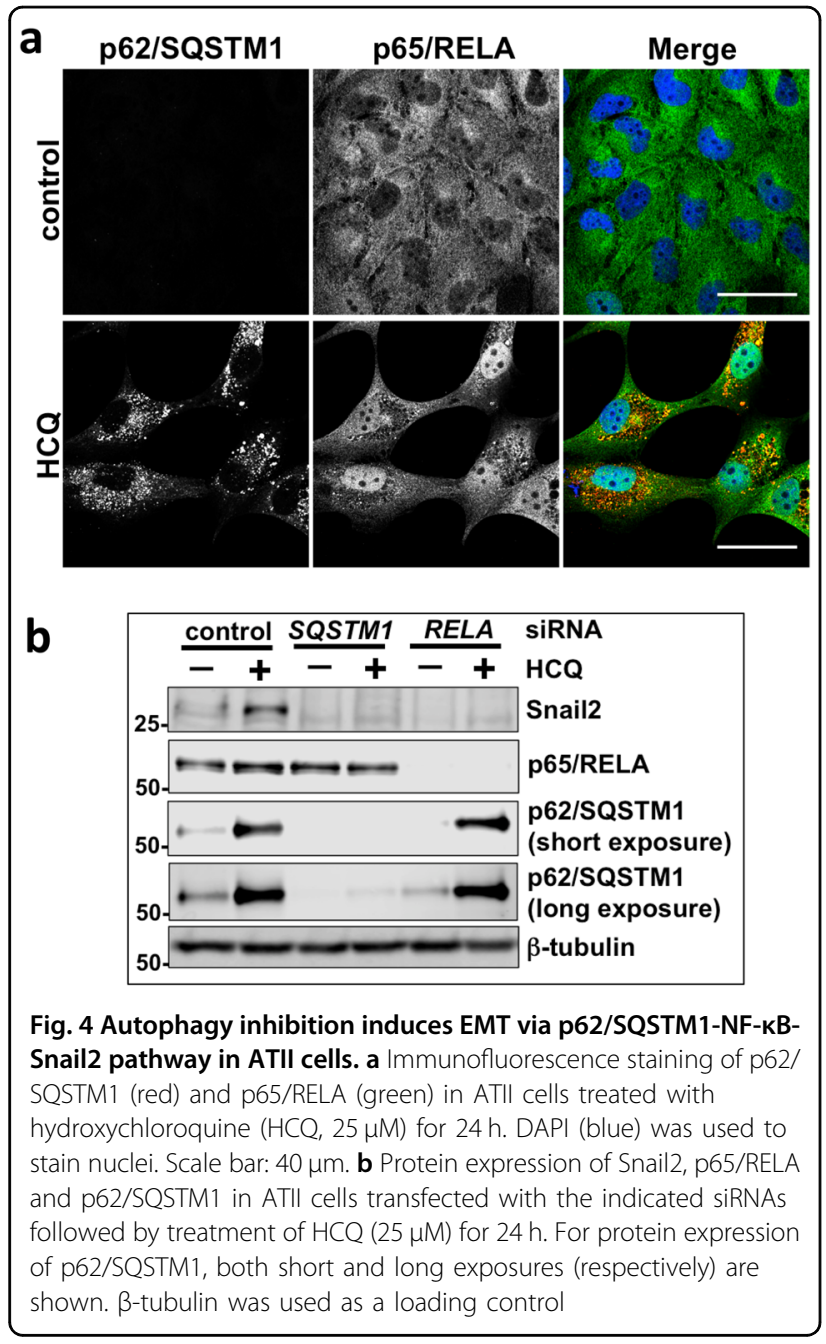

ATII cells undergoing autophagy inhibition-induced EMT induce fibroblast activation via Snail2-regulated paracrine signalling

Given that autophagy inhibition is able to induce EMT in ATII cells, we wanted to determine the role of these cells in the context of fibrosis, and whether they were contributing to the fibroblast population directly. Comparison of ECM components in control or ATG5 siRNA ATII cells demonstrated that ATII cells after autophagy inhibition-induced EMT did not express significantly more ECM genes (Supplementary Fig. S6). This suggested ECM production in IPF lung tissue (Fig. 1) was unlikely due directly to EMT but rather epithelial cells exhibiting an indirect effect on fibrogenesis. Therefore, we investigated whether ATG5-depleted ATII cells could produce secreted factors that activate fibroblasts. We treated primary human lung fibroblasts from IPF patients (IPF fibroblasts, IPFFs) with conditioned media (CM) from ATII cells transfected with control or ATG5 siRNA (Fig. 5a) without or with addition of transforming growth factor- $\beta$ (TGF- $\beta$ ), and assessed levels of $\alpha$-smooth muscle actin ( $\alpha$-SMA), a marker of myofibroblast differentiation. CM from ATII cells transfected with ATG5 siRNA without TGF- $\beta$ had minimal effect on the activation of fibroblasts (Fig. 5b). However in IPFFs, CM from ATG5depleted ATII cells together with TGF- $\beta$ achieved a synergistic effect in activating fibroblasts as assessed by $\alpha$ SMA protein levels (Fig. 5b). Notably, levels of phosphoSmad2 (p-Smad2) did not significantly change between different CM with TGF- $\beta$ treatment, suggesting $\alpha$-SMA increase may be Smad2-independent (Fig. 5b). Given the importance of Snail2 (SNAI2) in mediating EMT by autophagy inhibition (Fig. 5a), we hypothesised that Snail2 (SNAI2) may facilitate crosstalk by mediating paracrine signalling from ATII cells undergoing autophagy inhibition-induced EMT. Snail2 (SNAI2) depletion (Fig. 5a) in ATII cells eliminated the effects of CM from ATG5depleted ATII cells on activation of fibroblasts in the presence of TGF- $\beta$ (Fig. $5 \mathrm{c}$ ), highlighting the importance of Snail2 (SNAI2) as a key regulator of paracrine signalling between alveolar cells and fibroblasts in IPF under conditions where autophagy is defective.

\section{Discussion}

Fibrotic diseases are poorly characterised and lack effective treatment ${ }^{25}$, with fibrosis presenting as a pathological feature in a range of diseases affecting many organs ${ }^{25,26}$. A rise in the incidence of some fibrotic diseases ${ }^{1,3}$ and with fibrogenesis being accepted as a major cause of death ${ }^{27}$; it is evident that they are becoming an increasing healthcare burden, requiring further research to elucidate underlying mechanisms which drive fibrogenesis. IPF is an agingassociated chronic, interstitial lung disease ${ }^{2,28-34}$. Treatment options for patients are limited ${ }^{35}$ and the underlying mechanisms for fibrosis are still debated ${ }^{36}$. EMT has been suggested to have a direct role in IPF, with studies showing co-localisation of epithelial and mesenchymal markers ${ }^{37-41}$, and laser capture micro-dissection isolated RNA from epithelial cells in IPF lungs confirmed expression of mesenchymal markers ${ }^{42}$. However, lineage tracing studies found the number of fibroblasts derived from epithelial cells to be small $^{43,44}$, and these cells were not found to colocalise with $\alpha$-SMA suggesting they did not transition to myofibroblasts ${ }^{45,46}$. It also been proposed in a number of systems that epithelial cells that have undergone EMT may secrete a range of fibrogenic growth factors and cytokines, with impaired epithelial repair leading to aberrant epithelial-mesenchymal communication contributing to the recruitment and activation of myofibroblasts ${ }^{18,47,48}$. Here, we show that autophagy inhibition in ATII cells can induce EMT, and this contributes to fibrosis via aberrant epithelial-fibroblast crosstalk, rather than as a direct contribution to the fibroblast population. 

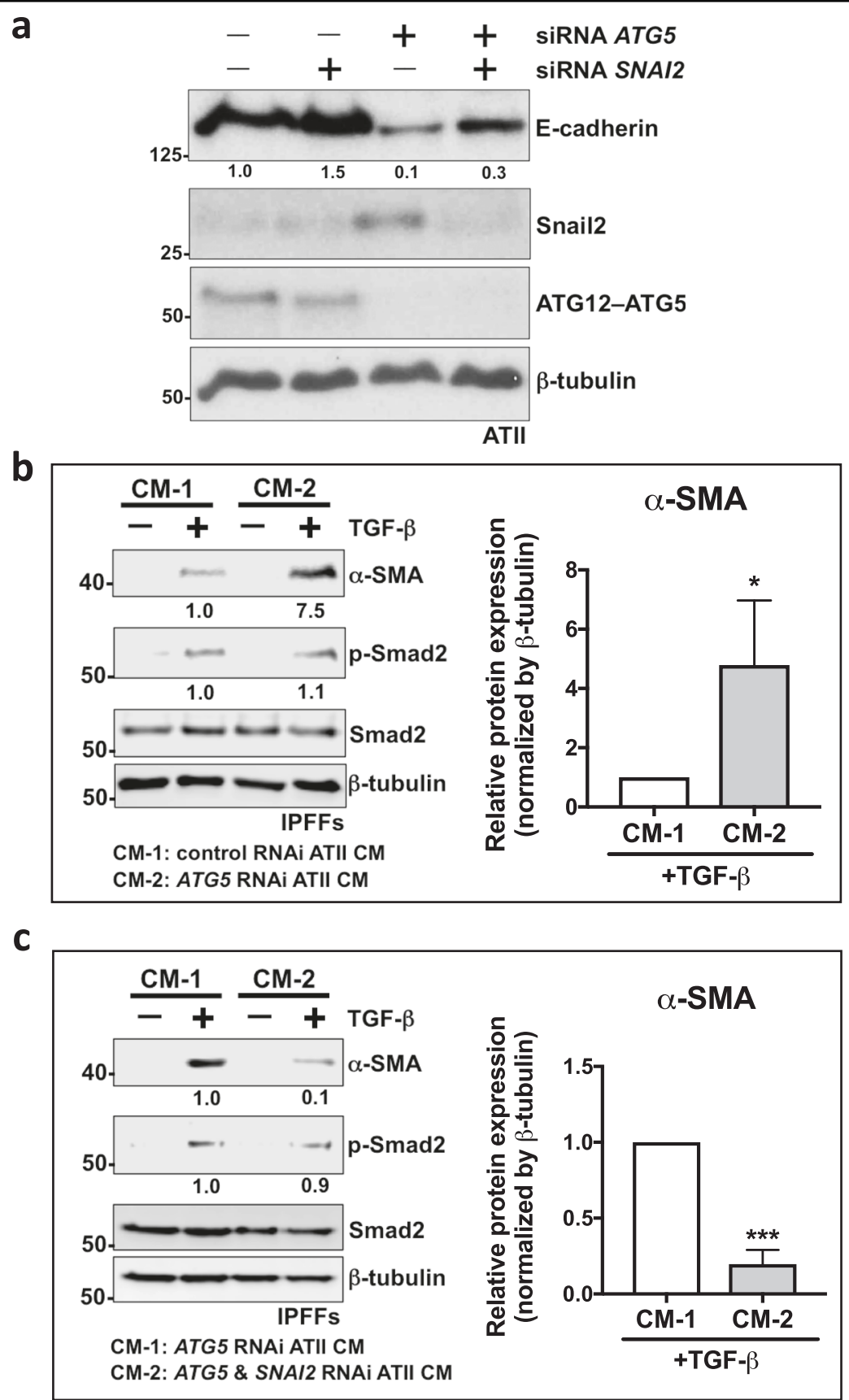

Fig. 5 ATIl cells undergoing autophagy inhibition-induced EMT induce fibroblast activation via Snail2-regulated paracrine signalling. a Protein expression of E-cadherin, ATG12-ATG5 and Snail2 in ATII cells transfected with indicated siRNAs. $\beta$-tubulin was used as a loading control. b, $\mathbf{c}$ Protein expression of a-SMA, Smad2 and phospho-Smad2 (p-Smad2) in IPFFs with indicated treatments. $\beta$-tubulin was used as a loading control. Scores under the bands are relative levels when compared with indicated controls (1.0). The graphs show relative a-SMA protein levels evaluated with ImageJ, and data are mean \pm s.d. $n=3$. ${ }^{*} P<0.05$. ${ }^{* *} P<0.001$

Autophagy is a tightly controlled, evolutionarily conserved biological process where long-lived proteins and damaged organelles are degraded. Manipulation of autophagy is now being utilised as a therapeutic approach in a number of fields, such as, neurodegenerative diseases and cancer ${ }^{49,50}$. On the other hand, decreased autophagic activity has been reported in many human diseases ${ }^{51}$, including $\mathrm{IPF}^{5-8}$. In IPF, ageing is a main risk factor in its development with patients under the age of 50 being $\operatorname{rare}^{29}$. Ageing has been linked to reduced autophagy in many contexts, including $\mathrm{IPF}^{5,52}$. Consistent with these findings ${ }^{6,8}$ we demonstrated strong immunostaining for 
p62/SQSTM1 in IPF epithelial cells of thickened alveolar septae whilst identifying only very weak signals in the control lung. We found significantly lower levels of SQSTM1 (p62) mRNA in IPF epithelial cells compared to control epithelial cells, which indicated that the increase in p62/SQSTM1 protein levels was due to reduced autophagic activity. Taken with previous findings ${ }^{6,8}$, these results indicate that autophagic activity is downregulated in IPF epithelial cells.

Whilst the concept of EMT is well established in the context of embryonic development, it also plays a role in in wound healing, cancer metastasis and fibrosis ${ }^{12}$. EMT requires a complex orchestration of multiple signalling pathways, including TGF- $\beta$, fibroblast growth factor, $\mathrm{Wnt} / \beta$-catenin, epidermal growth factor (EGF) and others. Loss of E-cadherin is considered to be a fundamental event in EMT. Snail1/2, ZEB1/2 and some basic helixloop-helix factors are potent repressors of E-cadherin expression $^{53,54}$. The role of EMT in cancer is detrimental whereas in wound healing, EMT as a response to injury can be beneficial, however, if the wound healing process is exaggerated it may lead to fibrosis. The interplay between autophagy and EMT have been reported in other disease contexts $^{55-57}$, with a recent study demonstrating inhibition of autophagy induces EMT via p62/SQSMT1-NFkB/ RELA pathway in RAS-mutated cancer cells ${ }^{24}$. This is mainly via upregulation of ZEB1 and to a lesser extent via Snail ${ }^{24}$. In contrast, in lung alveolar epithelial cells, autophagy inhibition induces EMT in the absence of RAS activation and is exclusively via up-regulation of Snail2 (Fig. 6a), indicating this effect is likely dependent on the cellular context. The importance of Snail2 in IPF has been demonstrated with Snail2 being upregulated in IPF lung epithelial cells, but not Snail1 or TWIST1, compared to control epithelial cells ${ }^{16,18}$. Jayachandran et al ${ }^{54}$ reported that Snail2-mediated EMT may contribute to the fibroblast pool. However, in this study, we found ATII cells undergoing autophagy inhibition-induced EMT induce fibroblast activation via Snail2-regulated paracrine signalling.

In IPF it was recently reported that micro-injuries could lead to the activation of the EGFR-RAS-ERK pathway, and ZEB1 was demonstrated to mediate paracrine signalling ${ }^{18}$. Similarly in renal fibrosis, tubular epithelial cells are able to promote myofibroblast differentiation and fibrogenesis without directly contributing to the fibroblast population by relaying signals to the interstitium. For example, in a murine model, damage-mediated Snail1 reactivationinduced partial EMT and resulted in renal fibrosis, but these cells did not contribute to myofibroblast or interstitial cell population ${ }^{47}$. Conversely, EMT was inhibited and interstitial fibrosis attenuated upon conditional deletion of Snail1 or Twist $1^{48}$.

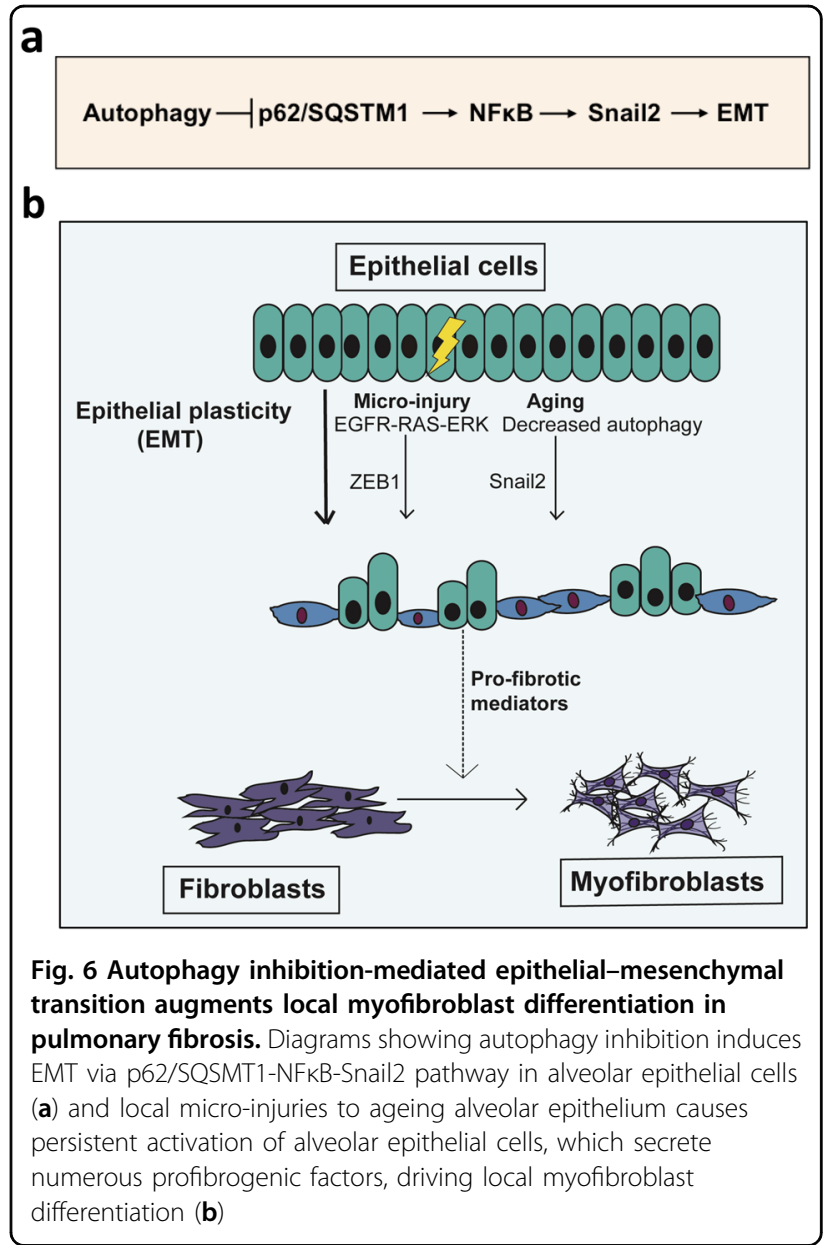

Taken together, we believe that repetitive local microinjuries to ageing alveolar epithelium cause persistent activation of alveolar epithelial cells, which secrete numerous profibrogenic factors, driving local myofibroblast differentiation (Fig. 6b). Thus, targeting of EMT inducers might have therapeutic potential in fibrotic conditions, with such therapies currently undergoing development in the context of malignancy ${ }^{58,59}$.

\section{Methods}

\section{Lung tissue sampling}

All human lung experiments were approved by the Southampton and South West Hampshire and the Mid and South Buckinghamshire Local Research Ethics Committees, and all subjects gave written informed consent. Clinically indicated IPF lung biopsy tissue samples and non-fibrotic control tissue samples (macroscopically normal lung sampled remote from a cancer site) were assessed as surplus to clinical diagnostic requirements. All IPF samples were from patients subsequently receiving a multidisciplinary diagnosis of IPF according to international consensus guidelines ${ }^{29}$. 


\section{Cell culture, reagents and transfections}

Primary parenchymal lung fibroblast cultures were established from IPF tissues as described previously ${ }^{60,61}$. Fibroblasts were cultured in Dulbecco's Modified Eagle's Medium (DMEM) supplemented with 10\% foetal bovine serum (FBS), 50 units/ml penicillin, $50 \mu \mathrm{g} / \mathrm{ml}$ streptomycin, $2 \mathrm{mM}$ L-glutamine, $1 \mathrm{mM}$ sodium pyruvate and $1 \times$ non-essential amino acids (DMEM/FBS) (all from Life Technologies). ATII cells ${ }^{18-20}$ were cultured in DCCM-1 (Biological Industries Ltd.) supplemented with 10\% NBCS (Life Technologies), 1\% penicillin, 1\% streptomycin and 1\% L-glutamine (all from Sigma Aldrich). A549 cells were cultured in DMEM (Fisher Scientific UK, 11594446) supplemented with 10\% FBS (Invitrogen) and antibiotics. All cells were kept at $37^{\circ} \mathrm{C}$ and $5 \% \mathrm{CO}_{2}$. For $3 \mathrm{D}$ culture, ATII cells were cultured as previously described ${ }^{62}$ in Matrigel (BD Biosciences). Bafilomycin-A1 (Baf-A1) was from Enzo Life Sciences. HCQ and Rapamycin were from Sigma Aldrich. No mycoplasma contamination was detected in the cell lines used.

siRNA oligos against ATG5 (MU-004374-04-0002), SQSTM1 (p62) (MU-010230-00-0002), SNAI2 (Snail2) (MU-017386-00-0002) and RELA (p65) (MU-003533-020002) were purchased from Dharmacon. Sequences are available from Dharmacon, or on request. As a negative control we used siGENOME RISC-Free siRNA (Dharmacon, D-001220-01). ATII and A549 cells were transfected with the indicated siRNA oligos at a final concentration of $35 \mathrm{nM}$ using DharmaFECT 2 reagent (Dharmacon).

\section{Western blot analysis}

Western blot analysis was performed with lysates from cells with urea buffer (8 M Urea, $1 \mathrm{M}$ Thiourea, $0.5 \%$ CHAPS, $50 \mathrm{mM}$ DTT, and $24 \mathrm{mM}$ Spermine). Primary antibodies were from: Santa Cruz ( $\beta$-actin, sc-47778; Ecadherin, sc-21791; Snail2, sc-10436), Abcam ( $\beta$-tubulin, ab6046), Cell Signalling Technology ( $\alpha$-SMA, 14968; Snail2, 9585; Smad2, 5339; Phospho-Smad2, 3104; $\beta$-tubulin, 86298; LC3, 2775; p62/SQSTM1, 5114; p65/ RELA, 8242; ATG5, 2630; mTOR, 2972; Phospho-mTOR Ser2448, 5536), and BD Transduction Laboratories (Ecadherin, 610405). Signals were detected using an ECL detection system (GE Healthcare) or Odyssey imaging system (LI-COR), and evaluated by ImageJ 1.42q software (National Institutes of Health).

\section{qRT-PCR}

Total RNA was isolated using RNeasy mini kit (Qiagen) according to manufacturer's instructions and quantified using a Nanodrop Spectophotometer 2000c (Thermo Fisher Scientific). Real-time quantitative RT-PCR was carried out using gene-specific primers (QuantiTect Primer Assays, Qiagen) for CDH1 (E-cadherin) (QT00080143), SNAI1 (Snail1) (QT00010010), SNAI2
(Snail2) (QT00044128), ZEB1 (QT00008555), ZEB2 (QT00008554), TWIST1 (QT00011956), COL1A1 (QT00037793), ACTA2 ( $\alpha$-SMA) (QT00088102), COL3A1 (QT00058233), FN1 (QT00038024), VIM (QT00095795), GAPDH (QT01192646) or ACTB ( $\beta$-actin) (QT01680476) with QuantiNova SYBR Green RT-PCR kits (Qiagen). Relative transcript levels of target genes were normalised to GAPDH or ACTB ( $\beta$-actin).

\section{Immunofluorescence microscopy}

Cells were fixed in $4 \%$ phosphate-buffered saline (PBS)-paraformaldehyde for $15 \mathrm{~min}$, incubated in $0.1 \%$ Triton X-100 for $5 \mathrm{~min}$ on ice, then in $0.2 \%$ fish skin gelatine in PBS for $1 \mathrm{~h}$ and stained for $1 \mathrm{~h}$ with an anti-Ecadherin (1:100, BD Biosciences, 610182) or anti-p62/ SQSTM1 (1:100, BD Biosciences, 610833) or anti-Snail2 (1:100, Cell Signalling Technology, 9585), p65/RELA (1:100, Cell Signalling Technology, 8242). Protein expression was detected using Alexa Fluor (1:400, Molecular Probes) for $20 \mathrm{~min}$. DAPI or TO-PRO-3 (Invitrogen) was used to stain nuclei $(1: 1000)$. Rhodamine phalloidin was used to visualise filamentous actin (F-actin) (Molecular Probes). For immunofluorescence staining of 3D cultures from ATII cells, spheres were fixed with $4 \%$ PBS-paraformaldehyde for $40 \mathrm{~min}$, permeabilised in $0.5 \%$ Triton X-100 for $10 \mathrm{~min}$ on ice and stained with rhodamine phalloidin for $1 \mathrm{~h}$ at room temperature. Spheres were counterstained with DAPI. Samples were observed using a confocal microscope system (Leica SP8). Acquired images were analysed using Photoshop (Adobe Systems) according to the guidelines of the journal.

\section{Wound-healing migration assay}

The wound-healing migration assays were done in conjunction with siRNA transfections in ATII cells. Seventy-two hours after siRNA transfections, confluent monolayers of cells were wounded with a p20 pipette tip (time 0). Phase-contrast images were taken using an Olympus inverted microscope at time 0 or $20 \mathrm{~h}$ after the scratch wound, invasive cells were evaluated by ImageJ software (National Institutes of Health).

\section{Transwell migration and Matrigel invasion assay}

For the Transwell migration assay, Transwell membranes $(8-\mu \mathrm{m}$ pore size, $6.5-\mathrm{mm}$ diameter; Corning Costar, 3422) were used. The bottom chambers of the Transwell were filled with migration-inducing medium (with 50\% FBS). The top chambers were seeded with $1.5 \times$ $10^{5}$ live serum-starved control or ATG5-depleted ATII cells per well. After $24 \mathrm{~h}$, the filters were fixed with $4 \%$ paraformaldehyde for $10 \mathrm{~min}$ at room temperature; subsequently, the cells on the upper side of the membrane were scraped with a cotton swab. Similar inserts coated with Matrigel (Corning, 354480) were used to determine 
invasive potential in invasion assays. Filters were stained with crystal violet for light microscopy. Images were taken using an Olympus inverted microscope and migratory cells were evaluated by ImageJ $1.42 \mathrm{q}$ software (National Institutes of Health).

\section{Immunohistochemistry, haematoxylin and eosin (H/E) and tinctorial stains}

Control or IPF lung tissues were fixed and embedded in paraffin wax; tissue sections $(4 \mu \mathrm{m})$ were processed and stained as previously described ${ }^{18}$. Briefly, the tissue sections were de-waxed, rehydrated and incubated with 3\% hydrogen peroxide in methanol for $10 \mathrm{~min}$ to block endogenous peroxidase activity. Sections were then blocked with normal goat serum and incubated at room temperature with a primary antibody against p62/ SQSTM1 (1:100, Progen, GP62_C), followed by a biotinylated secondary antibody (1:500, Vector Laboratories Ltd., UK); antibody binding was detected using streptavidin-conjugated horse-radish peroxidase and visualised using DAB (DAKO) before counterstaining with Mayer's Haematoxylin. For H/E stain, Shandon Varistain 24-4 automatic slide stainer (Thermo Fisher Scientific) was used. For tinctorial stain, Trichrome stain (Abcam ab150686) was used according to the manufacturers' instructions. Images were acquired using an Olympus Dotslide Scanner VS110.

\section{Statistical analysis and repeatability of experiments}

Each experiment was repeated at least twice. Unless otherwise noted, data are presented as mean and s.d., and a two-tailed, unpaired or paired Student's $t$ test was used to compare two groups for independent samples. $P<0.05$ was considered statistically significant.

\section{Acknowledgements}

This project was supported by an Academy of Medical Sciences/the Wellcome Trust Springboard Award [SBF002/1038], the Wessex Medical Trust and AAIR Charity. C.H. was supported by Gerald Kerkut Charitable Trust and University of Southampton Central VC Scholarship Scheme. D.L. was supported by the National Natural Science Foundation of China [81772827]. F.C. was supported by Medical Research Foundation [MRF-091-0003-RG-CONFO]. L.Y. was supported by China Scholarship Council. Y.Z. was supported by an Institute for Life Sciences PhD Studentship. A.J.S. was supportd by Bloodwise grant 12044. J.D. was supported by the Francis Crick Institute which receives its core funding from Cancer Research UK (FC001070), the UK Medical Research Council (FC001070) and the Wellcome Trust (FC001070). X.L. was supported by the Ludwig Institute for Cancer Research and the NIHR Oxford Biomedical Research Centre. M.G.J. was supported by the Wellcome Trust [100638/Z/12/Z]. We thank Carine Fixmer, Maria Lane, Benjamin Johnson and the nurses of the Southampton NIHR Biomedical Research Unit for their help in the collection of human samples, supported by the Wessex Clinical Research Network and the National Institute of Health Research, UK.

\section{Author details}

${ }^{1}$ Biological Sciences, Faculty of Environmental and Life Sciences, University of Southampton, Southampton SO17 1BJ, UK. ${ }^{2}$ Department of Oncology, Tongji Hospital, Tongji Medical College, Huazhong University of Science and Technology, Wuhan, China. ${ }^{3}$ Clinical and Experimental Sciences, Faculty of Medicine, University of Southampton, Southampton SO16 6YD, UK. ${ }^{4} \mathrm{NIHR}$
Southampton Biomedical Research Centre, University Hospital Southampton, Southampton SO16 6YD, UK. ${ }^{5}$ University Hospital Southampton, Southampton SO16 6YD, UK. ${ }^{6}$ Cancer Sciences Unit, University of Southampton, Somers Building, Southampton General Hospital, Southampton SO16 6YD, UK. ${ }^{7}$ Oncogene Biology, The Francis Crick Institute, London NW1 1AT, UK. ${ }^{8}$ Unità Operativa Complessa di Pneumologia, Università Cattolica del Sacro Cuore, Fondazione Policlinico A Gemelli IRCCS, Rome, Italy. ${ }^{9}$ Ludwig Institute for Cancer Research, Nuffield Department of Clinical Medicine, University of Oxford, Oxford OX3 7DQ, UK. ${ }^{10}$ Institute for Life Sciences, University of Southampton, Southampton SO17 1BJ, UK

\section{Conflict of interest}

The authors declare that they have no conflict of interest.

\section{Publisher's note}

Springer Nature remains neutral with regard to jurisdictional claims in published maps and institutional affiliations.

Supplementary Information accompanies this paper at (https://doi.org/ 10.1038/s41419-019-1820-x).

Received: 2 May 2019 Revised: 28 June 2019 Accepted: 18 July 2019 Published online: 07 August 2019

\section{References}

1. Hutchinson, J., Fogarty, A., Hubbard, R. \& McKeever, T. Global incidence and mortality of idiopathic pulmonary fibrosis: a systematic review. Eur. Respir. J. 46, 795-806 (2015).

2. Richeldi, L., Collard, H. R. \& Jones, M. G. Idiopathic pulmonary fibrosis. Lancet 389, 1941-1952 (2017).

3. Raghu, G., Weycker, D., Edelsberg, J., Bradford, W. Z. \& Oster, G. Incidence and prevalence of idiopathic pulmonary fibrosis. Am. J. Respir. Crit. Care Med. 174, 810-816 (2006).

4. Martinez-Lopez, N., Athonvarangkul, D. \& Singh, R. Autophagy and aging. Adv. Exp. Med. Biol. 847, 73-87 (2015)

5. Romero, Y. et al. mTORC1 activation decreases autophagy in aging and idiopathic pulmonary fibrosis and contributes to apoptosis resistance in IPF fibroblasts. Aging Cell 15, 1103-1112 (2016).

6. Patel, A. S. et al. Autophagy in idiopathic pulmonary fibrosis. PLOS ONE 7, e41394 (2012).

7. Rangarajan, S. et al. Novel mechanisms for the antifibrotic action of nintedanib. Am. J. Respir. Cell Mol. Biol. 54, 51-59 (2016).

8. Araya, J. et al. Insufficient autophagy in idiopathic pulmonary fibrosis. Am. J. Physiol. Lung Cell Mol. Physiol. 304, L56-L69 (2013).

9. Ghavami, S. et al. Autophagy and the unfolded protein response promote profibrotic effects of TGF- $\beta_{1}$ in human lung fibroblasts. Am. J. Physiol. Cell Mol. Physiol. 314, L493-L504 (2018).

10. Bento, C. F. et al. Mammalian autophagy: how does it work? Annu Rev. Biochem. 85, 685-713 (2016).

11. Singh, K. K. et al. The essential autophagy gene ATG7 modulates organ fibrosis via regulation of endothelial-to-mesenchymal transition. J. Biol. Chem. 290, 2547-2559 (2015).

12. Nieto, A., Huang, R., Jackson, R. \& Thiery, J. EMT: 2016. Cell 166, 21-45 (2016).

13. Peinado, H., Olmeda, D. \& Cano, A. Snail, Zeb and bHLH factors in tumour progression: an alliance against the epithelial phenotype? Nat. Rev. Cancer $\mathbf{7}$, 415-428 (2007).

14. Nieto, M. A. The ins and outs of the epithelial to mesenchymal transition in health and disease. Annu Rev. Cell Dev. Biol. 27, 347-376 (2011).

15. Rusten, T. E. \& Stenmark, H. p62, an autophagy hero or culprit? Nat. Cell Biol. 12, 207-209 (2010).

16. $\mathrm{Xu}, \mathrm{Y}$. et al. Single-cell RNA sequencing identifies diverse roles of epithelial cells in idiopathic pulmonary fibrosis. JCl Insight 1, e90558 (2017).

17. Moscat, J. \& Diaz-Meco, M. T. p62 at the crossroads of autophagy, apoptosis, and cancer. Cell 137, 1001-1004 (2009).

18. Yao, $L$. et al. Paracrine signalling during ZEB1-mediated epithelial-mesenchymal transition augments local myofibroblast differentiation in lung fibrosis. Cell Death Differ. 26, 943-957 (2019). 
19. Molina-Arcas, M., Hancock, D. C., Sheridan, C., Kumar, M. S. \& Downward, J. Coordinate direct input of both KRAS and IGF1 receptor to activation of PI3 kinase in KRAS-mutant lung cancer. Cancer Disco. 3, 548-563 (2013).

20. Coelho, M. A. et al. Oncogenic RAS signaling promotes tumor immunoresistance by stabilizing PD-L1 mRNA. Immunity 47, 1083-1099.e6 (2017).

21. DeVorkin, L. \& Lum, J. J. In: Strategies to block autophagy in tumor cells. Autophagy: Cancer, Other Pathologies, Inflammation, Immunity, Infection, and Aging. (ed. Hayat, M.) 121-130 (Academic Press: New York, 2014).

22. Thiery, J. P. \& Sleeman, J. P. Complex networks orchestrate epithelial-mesenchymal transitions. Nat. Rev. Mol. Cell Biol. 7, 131-142 (2006)

23. Ballou, L. M. \& Lin, R. Z. Rapamycin and mTOR kinase inhibitors. J. Chem. Biol. 1 27-36 (2008).

24. Wang, Y. et al. Autophagy inhibition specifically promotes epithelialmesenchymal transition and invasion in RAS-mutated cancer cells. Autophagy. 15, 886-899 (2019).

25. Thannickal, V. J., Zhou, Y., Gaggar, A. \& Duncan, S. R. Fibrosis: ultimate and proximate causes. J. Clin. Invest. 124, 4673-4677 (2014).

26. Wynn, T. A. Fibrotic disease and the $\mathrm{T}(\mathrm{H}) 1 / \mathrm{T}(\mathrm{H}) 2$ paradigm. Nat. Rev. Immunol. 4, 583-594 (2004).

27. Wynn, T. A. \& Ramalingam, T. R. Mechanisms of fibrosis: therapeutic translation for fibrotic disease. Nat. Med. 18, 1028-1040 (2012).

28. Pardo, A. \& Selman, M. Lung fibroblasts, aging, and idiopathic pulmonary fibrosis. Ann. Am. Thorac. Soc. 13(Suppl 5), S417-S421 (2016).

29. Raghu, G. et al. Diagnosis of idiopathic pulmonary fibrosis. An Official ATS/ERS/ JRS/ALAT Clinical Practice Guideline. Am. J. Respir. Crit. Care Med. 198, e44-e68 (2018).

30. Minagawa, S. et al. Accelerated epithelial cell senescence in IPF and the inhibitory role of SIRT6 in TGF- $\beta$-induced senescence of human bronchial epithelial cells. Am. J. Physiol. Cell Mol. Physiol. 300, L391-L401 (2011).

31. Tsakiri, K. D. et al. Adult-onset pulmonary fibrosis caused by mutations in telomerase. Proc. Natl Acad. Sci. USA 104, 7552-7557 (2007).

32. Alder, J. K. et al. Short telomeres are a risk factor for idiopathic pulmonary fibrosis. Proc. Natl Acad. Sci. USA 105, 13051-13056 (2008).

33. Bueno, M. et al. PINK1 deficiency impairs mitochondrial homeostasis and promotes lung fibrosis. J. Clin. Invest. 125, 521-538 (2015).

34. Fingerlin, T. E. et al. Genome-wide association study identifies multiple susceptibility loci for pulmonary fibrosis. Nat. Genet. 45, 613-620 (2013).

35. Spagnolo, P., Tzouvelekis, A. \& Bonella, F. The management of patients with idiopathic pulmonary fibrosis. Front. Med. 5, 148 (2018).

36. Hill, C., Jones, M. G., Davies, D. E. \& Wang, Y. Epithelial-mesenchymal transition contributes to pulmonary fibrosis via aberrant epithelial/fibroblastic cross-talk J. Lung Health Dis. 3, 31-35 (2019).

37. Chilosi, M. et al. Epithelial to mesenchymal transition-related proteins ZEB1, $\beta$-catenin and $\beta$-tubulin-III in idiopathic pulmonary fibrosis. Mod. Pathol. 30 26-38 (2017).

38. Park, J. S. et al. Clinical significance of mTOR, ZEB1, ROCK1 expression in lung tissues of pulmonary fibrosis patients. BMC Pulm. Med. 14, 168 (2014).

39. Lomas, N. J., Watts, K. L., Akram, K. M., Forsyth, N. R. \& Spiteri, M. A. Idiopathic pulmonary fibrosis: immunohistochemical analysis provides fresh insights into lung tissue remodelling with implications for novel prognostic markers. Int J. Clin. Exp. Pathol. 5, 58-71 (2012).

40. Willis, B. C. et al. Induction of epithelial-mesenchymal transition in alveolar epithelial cells by transforming growth factor-beta1: potential role in idiopathic pulmonary fibrosis. Am. J. Pathol. 166, 1321-1332 (2005).
41. Harada, T. et al. Epithelial-mesenchymal transition in human lungs with usual interstitial pneumonia: quantitative immunohistochemistry. Pathol. Int. 60, 14-21 (2010).

42. Marmai, C. et al. Alveolar epithelial cells express mesenchymal proteins in patients with idiopathic pulmonary fibrosis. AJP Lung Cell Mol. Physiol. 301, L71-L78 (2011).

43. Rock, J. R. et al. Multiple stromal populations contribute to pulmonary fibrosis without evidence for epithelial to mesenchymal transition. Proc. Natl Acad. Sci. 108, E1475-E1483 (2011).

44. Humphreys, B. D. et al. Fate tracing reveals the pericyte and not epithelial origin of myofibroblasts in kidney fibrosis. Am. J. Pathol. 176, 85-97 (2010).

45. Tanjore, $H$. et al. Contribution of epithelial-derived fibroblasts to bleomycininduced lung fibrosis. Am. J. Respir. Crit. Care Med. 180, 657-665 (2009).

46. Degryse, A. L. et al. TGF $\beta$ signaling in lung epithelium regulates bleomycininduced alveolar injury and fibroblast recruitment. Am. J. Physiol. Lung Cell Mol. Physiol. 300, L887-L897 (2011).

47. Grande, M. T. et al. Snail1-induced partial epithelial-to-mesenchymal transition drives renal fibrosis in mice and can be targeted to reverse established disease. Nat. Med. 21, 989-997 (2015).

48. Lovisa, S. et al. Epithelial-to-mesenchymal transition induces cell cycle arrest and parenchymal damage in renal fibrosis. Nat. Med. 21, 998-1009 (2015).

49. Harris, H. \& Rubinsztein, D. C. Control of autophagy as a therapy for neurodegenerative disease. Nat. Rev. Neurol. 8, 108-117 (2012).

50. White, E. Deconvoluting the context-dependent role for autophagy in cancer. Nat. Rev. Cancer 12, 401-410 (2012).

51. Saha, S., Panigrahi, D. P., Patil, S. \& Bhutia, S. K. Autophagy in health and disease: a comprehensive review. Biomed. Pharm. 104, 485-495 (2018).

52. Rubinsztein, D. C., Mariño, G. \& Kroemer, G. Autophagy and aging. Cell 146, 682-695 (2011)

53. Lamouille, S., Xu, J. \& Derynck, R. Molecular mechanisms of epithelial-mesenchymal transition. Nat. Rev. Mol. Cell Biol. 15, 178-196 (2014).

54. Jayachandran, $A$. et al. SNAl transcription factors mediate epithelialmesenchymal transition in lung fibrosis. Thorax 64, 1053-1061 (2009).

55. Catalano, M. et al. Autophagy induction impairs migration and invasion by reversing EMT in glioblastoma cells. Mol. Oncol. 9, 1612-1625 (2015).

56. Wei, R. et al. FAT4 regulates the EMT and autophagy in colorectal cancer cells in part via the PI3K-AKT signaling axis. J. Exp. Clin. Cancer Res. 38, 112 (2019).

57. Gugnoni, M., Sancisi, V., Manzotti, G., Gandolfi, G. \& Ciarrocchi, A. Autophagy and epithelial-mesenchymal transition: an intricate interplay in cancer. Cell Death Dis. 7, e2520 (2016).

58. Sakata, J. et al. Inhibition of ZEB1 leads to inversion of metastatic characteristics and restoration of paclitaxel sensitivity of chronic chemoresistant ovarian carcinoma cells. Oncotarget 8, 99482-99494 (2017).

59. Kothari, A., Mi, Z., Zapf, M. \& Kuo, P. Novel clinical therapeutics targeting the epithelial to mesenchymal transition. Clin. Transl. Med. 3, 35 (2014).

60. Conforti, F. et al. The histone deacetylase inhibitor, romidepsin, as a potential treatment for pulmonary fibrosis. Oncotarget 8, 48737-48754 (2017).

61. Jones, M. G. et al. Nanoscale dysregulation of collagen structure-function disrupts mechano-homeostasis and mediates pulmonary fibrosis. Elife $\mathbf{7}$ e36354 (2018).

62. Yu, W. et al. Formation of cysts by alveolar type II cells in three-dimensional culture reveals a novel mechanism for epithelial morphogenesis. Mol. Biol. Cell 18, 1693-1700 (2007) 